\title{
Spontaneous Tuberculous Enterocutaneous Fistula at Iliac Crest - A Rare Case
}

\section{Authors \\ Girish D Bakhshi, Kavita V Jadhav, Dayanand D Choure, Mukund B Tayade, Palak Jaiswal, Apurva Anand}

Department of General Surgery, Grant Government Medical College \& Sir J.J. Group of Hospitals, Byculla, Mumbai-08

Corresponding Author

Dr Girish D. Bakhshi

Devneeti, Plot-61, Sector-7, Koper Khairane, Navi Mumbai-400709, Maharashtra, India Email:- gdbakhshi@yahoo.com,Tel : +91-9820218198

ABSTRACT
Enterocutaneous fistula is an abnormal communication between bowel and skin. Most cases of
enterocutaneous fistula (ECF) are iatrogenic and develop following abdominal surgeries. Other common
causes are inflammatory bowel disease, malignancy, post radiation therapy, anastomotic breakdown. Less
commonly, ECFs develop spontaneously as part of the disease process in Crohn's disease, diverticulitis,
tuberculosis or enteric fever. We present a case of spontaneous enterocutaneous fistula due to
tuberculosis at right iliac creast region posteriorly near ilial tubercle in flank, an uncommon site.
KEY WORDS Spontaneous ECF, Iliac creast-a rare site ECF

\section{INTRODUCTION}

Fistula is an abnormal communication between two epithelial surfaces. Enterocutaneous fistula (ECF) is an abnormal communication between the bowel and skin. Spontaneous ECF are rare and common and may develope due to Crohn's disease, diverticulitis, tuberculosis. Spontaneous ECF without prior history of tuberculosis or without any surgical intervention of bowel in past is rare with tuberculosis. Even if spontaneous ECF develops, it commonly occurs over anterior abdominal wall or scar site. In present case, Spontaneous ECF developed in iliac crest region posteriorly in flank above \& lateral to ilial tubercle. It is rare site of Spontaneous ECF.

\section{CASE SUMMARY}

A 65 years old male presented with history of small swelling in right lumbar region just above right iliac crest region at ilial tubercle since 7days. It burst open spontaneously with feculent discharge since 1 day. Patient had no history of pain in abdomen, vomitings, constipation, trauma, surgery, radiation or history suggestive of inflammatory bowel disease. Patient had no past history suggestive of tuberculosis or immunocompromised state.

On examination, patient was afebrile and vitally stable. Abdomen was soft, nontender on palpation. Small fistulous opening in the right flank just superior to right iliac crest near ilial tubercle 
region was found. Feculent discharge was present through fistula and quantity of it was less than $100 \mathrm{cc} /$ day. Excoriation of surrounding skin was present.

On investigation, routine blood investigation and erythrocyte sedimentation rate (ESR) were within normal limit with montoux test negative. Local part ultrasound was suggestive of fistulous tract in right lumbar region communicating with underlying bowel. Barium enema and colonoscopy were normal ruling out any stricture. Xray Fistulogram was suggestive of passage of contrast with enhancement of whole large bowel (Fig.1). Computed tomography (CT) of abdomen with pelvis suggestive of eneterocutaneous fistulous tract with passage of contrast from caecum to iliac crest region (Fig.2a \& 2b).

Initially conservative line of management was given to patient with intravenous antibiotics, high protein diet and enemas. Patient did not respond to it. Hence, exploratory laparotomy was performed. Intraoperatively there was no evidence of bowel thickening, stricture, mesenteric lymph nodes. Fistulous tract was found communicating between caecum and skin with caecum densly adhered to lateral wall. Right hemicolectomy with excision of fistulous tract was done( Fig.3a, 3b \& 3c).

Histopathology examination revealed granulomatous inflammation suggestive of tuberculosis. Post operatively patient was given anti kochs therapy (AKT) for 9 months Isoniazide, rifampicin, pyrizinamide, ethambutol (HRZE) for 3 months followed by Isoniazide, rifampicin (HR) for 6 months. Patient found to be completely asymptomatic and disease free after 1 year of follow up.

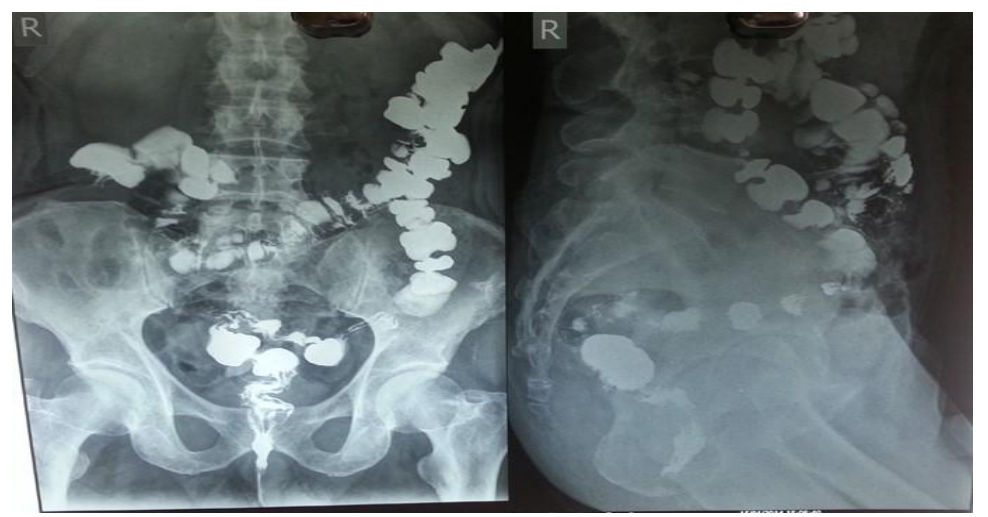

Fig. 1 - Barium Enema showing enhancement of whole colon

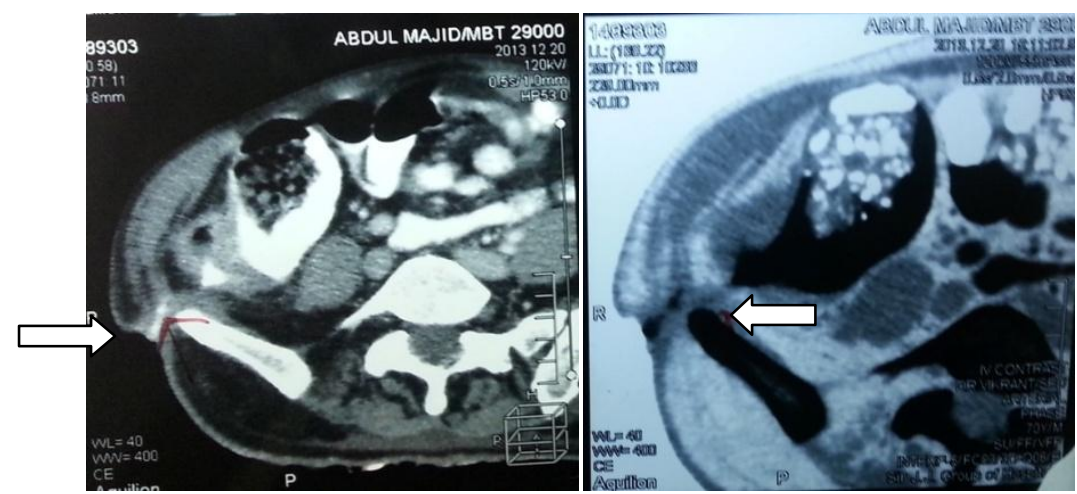

Fig 2a \& b Fig. 2a \& 2b - CT axial views showing eneterocutaneous fistulous tract with passage of contrast from caecum to iliac crest region as indicated by arrow 


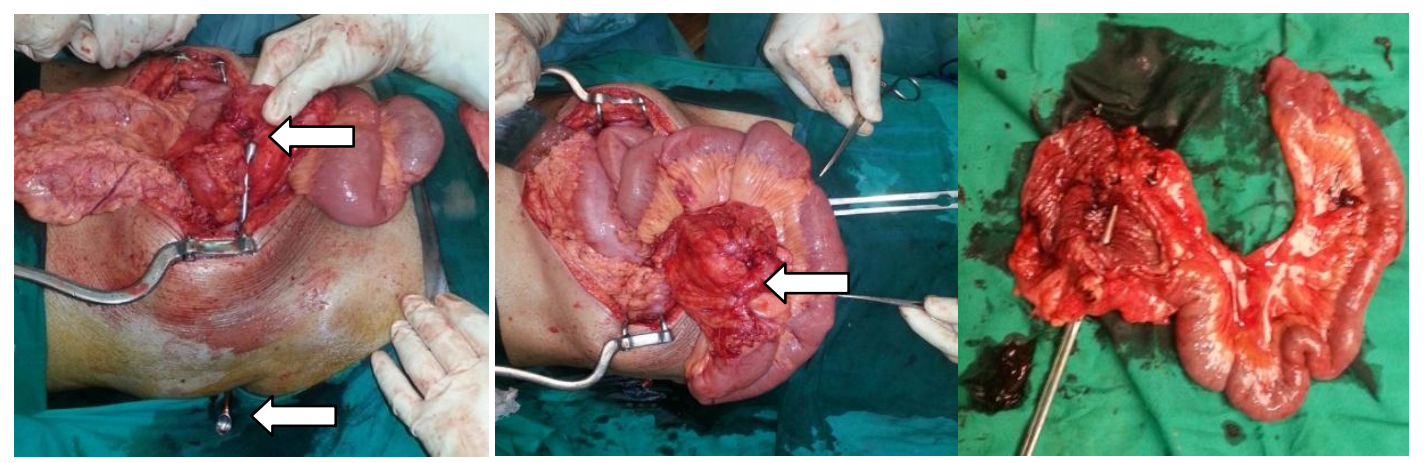

Fig. 3a - Intraoperative images showing ECF with internal \& external opening marked by arrows, 3b - ECF with Opening at caecum

3c - Right hemicolectomy resected specimen with internal opening of ECF at caecum

\section{DISCUSSION}

Spontaneous ECFs are uncommon in absence of prior surgery. It commonly developes due to malignancy, inflammatory bowel disease, tuberculosis, radiation enteritis, diverticular disease and rarely with enteric fever. More common with history of prior surgery. Anterior abdominal wall is the common site for occurrence of spontaneous ECF.

Tuberculosis is common disease in developing countries. It manifests in different ways. It almost affects all systems of human body. Abdominal tuberculosis is common amongst them. Rarely, this abdominal tuberculosis may present with ECF or spontaneous ECF.

Uptil now few rare site presentation of spontaneous tubercular ECF have been reported. Rao and Mitra et $\mathrm{al}^{(1)}$ reported 5 children with spontaneous tubercular enteroumbilical fistulas. Ameh and Awotula et $\mathrm{al}^{(2)}$ reported spontaneous scrotal faecal fistula in infants. Rai and Pahwa et $\mathrm{al}^{(3)}$ presented spontaneous ECF in right inguinal region.

In Present case, patient developed spontaneous ECF at right iliac crest region superior and lateral to ilial tubercle, a rare site for occurence of ECF.As it was through retroperitoneum, patient did not manifest signs of peritonitis. This was a localised fistula due to surrounding fibrotic reaction. This prevented sepsis in present case. As a result initially conservative management was given after ruling out distal obstruction.

In present case, patient had no history suggestive of any surgery or irradiation in past. Patient also had no history suggestive of tuberculosis, malignancy or inflammatory bowel disease. Patient presented as spontaneous enetrocutneous fistula involving only caecum with opening at upper border of iliac crest without any distal obstruction. This made this a rare presentation of tuberculosis \& rare site of enterocutaneous fistula formation. A surgical intervention with AKT gave complete relief to the pt.

\section{CONCLUSION}

Even after detailed clinical history of the patient regarding possible aetiologies for development of such rare site enterocutaneous fistula, we may not get certain cause. Conservative line of management may not cure such spontaneous ECF. Complete surgical excision of tract and resection and anastomosis of affected bowel is gold standard for nonresponding ECF. Suspicion of tuberculosis should be kept in mind even though history and routine investigations are not in favour of tuberculosis. Such rare site spontaneous enetrocutaneous fistula can occur due to tuberculosis. Postoperative AKT helps to prevent recurrance. 


\section{REFERRANCES}

1. Rao PL, Mitra SK, Pathak IC. Spontaneous tuberculous enteroumbilical fistulas. Am J Gastroentrol.1979;72:671-5.

2. Ameh EA, Awotula OP, Amoah JN. Spontaneous scrotal faecal fistula in infants. Pediatr Surg Int. 2002;18:524-5.

3. Rai \& Pahwa et al. Spontaneous Enterocutaneous Fistula - A Rare Presentation. Internet Journal of Surgery; $2009,19: 15$ 